\title{
Sistema de clasificación de deformaciones pédicas por procesamiento digital de imágenes y lógica difusa en LabVIEW
}

\author{
Héctor García Estrada ${ }^{1}$, Omar Alejandro Linares Escobar ${ }^{1}$, \\ Angelo Pastrana Manzanero ${ }^{1}$, Luis Carlos Martínez Ruiz ${ }^{1}$, \\ María Guadalupe Ramírez Sotelo ${ }^{2}$, Agustín Ignacio Cabrera Llanos ${ }^{1}$ \\ ${ }^{1}$ Instituto Politécnico Nacional, Unidad Profesional Interdisciplinaria de Biotecnología, \\ Departamento de Bioprocesos, Ciudad de México, México \\ ${ }^{2}$ Instituto Politécnico Nacional, Unidad Profesional Interdisciplinaria de Biotecnología, \\ Departamento de Bioingeniería, Ciudad de México, México \\ aicllbuda@yahoo.com
}

\begin{abstract}
Resumen. En el presente trabajo se muestra el desarrollo de un sistema de clasificación de malformaciones pédicas usando procesamiento digital de imágenes en LabVIEW. El sistema funciona en tres etapas: captura de las imágenes del pie, procesamiento de las imágenes y clasificación. Para la toma de las imágenes se utilizaron webcams colocadas en la base de un andador de hierro con superficie de acrílico, de manera que se obtiene la planta del pie. Posteriormente, se umbraliza la imagen adquirida usando el rango de intensidad en la zona donde se ubica la huella del pie de manera que se obtiene la superficie que hace contacto con el acrílico al momento de la pisada. A partir de la huella se obtienen las medidas de la longitud del arco y del ancho del pie, desde los cuales se calcula el porcentaje de relación entre estas mediciones. Finalmente, este porcentaje se ingresa a un sistema de lógica difusa, pudiendo clasificar las deformaciones en pie plano, normal, cavo, cavo fuerte y cavo extremo. Este sistema disminuye el tiempo de respuesta para clasificación de la deformación pédica por parte del especialista y permite el desarrollo de un sistema de apoyo al diagnóstico de la malformación.
\end{abstract}

Palabras clave: LabVIEW, procesamiento digital de imágenes, lógica difusa, diagnóstico por imagen, malformación pédica.

\section{Classification System of Pedic Deformations Using Digital Image Processing and Fuzzy Logic in LabVIEW}

\begin{abstract}
The present Project shows the development of a classification system for foot malformation using digital image processing in LabVIEW. The system Works in three stages: acquisition of the foot image, image processing and classification. For the image capture were used webcams at the base of an iron walker with acrylic surface, so that the sole of the foot is obtained. Later, the acquired image become binary by using thresholding by way of obtain the sole surface that makes contact with the acrylic surface at the moment of the footstep.
\end{abstract}




\begin{abstract}
From the footprint is obtain the measure of arc long and foot width from which the percentage of relationship between these measurements is calculated. Finally, this percentage enters to a fuzzy logic system being able to classify the deformations in flat foot, normal, cavus foot, strong cavus foot and high cavus foot. This system decreases the response time for classify the foot malformations given by the specialist and allows the development of a support system for malformation diagnostic.
\end{abstract}

Keywords: LabVIEW, digital image processing, fuzzy logic, diagnostic imaging, pedic malformation.

\title{
1. Introducción
}

\subsection{Malformaciones pédicas}

Una malformación es una anomalía morfológica, generalmente de origen congénito. En el caso de las malformaciones pédicas, estas se dan en la planta del pie, provocando que la huella se deforme alterando su biomecánica [1].

Las malformaciones se pueden clasificar por la forma en la que se aprecia la planta del pie, siendo las más comunes pie plano y pie cavo (Fig. 1). El pie plano se caracteriza por la ausencia de arco y una huella uniforme; el cavo presenta un arco muy pronunciado en la huella. La técnica más común para el diagnóstico de malformaciones pédicas es el fotopodograma, el cual consiste en obtener una imagen de la huella del pie para realizar mediciones desde las cuales se puede obtener una valoración por parte del especialista [2].

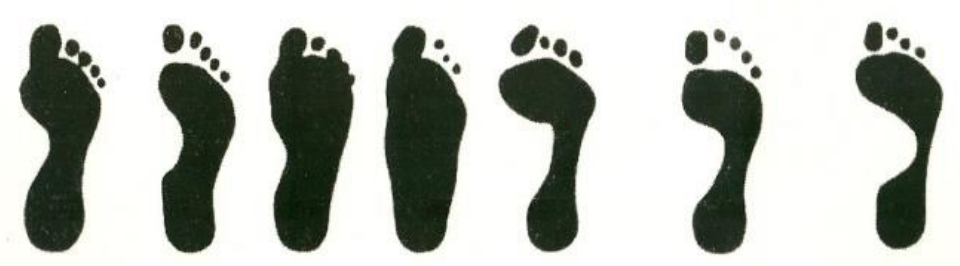

Fig. 1. Ejemplos de fotopodogramas de diferentes malformaciones.

\subsection{Procesamiento digital de imágenes}

El procesamiento digital de imágenes es una disciplina y un conjunto de técnicas para trabajar, analizar y obtener información de imágenes a través de medios electrónicos usando los valores numéricos de las matrices que componen las imágenes digitales, donde cada pixel corresponde a una posición de la matriz y cada valor numérico a una intensidad (Fig. 2) [3]. 

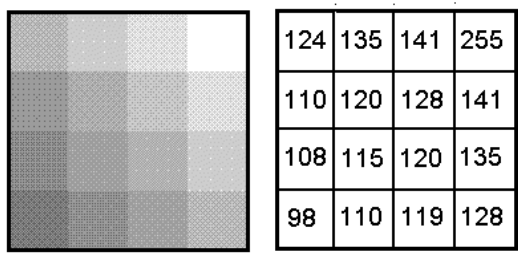

Fig. 2. Comparación de imagen digital con su imagen correspondiente.

Las técnicas del procesamiento digital de imágenes son muy variadas y permiten analizar diferentes tipos de información en las imágenes pasando de la determinación de un color a la detección de objetos [4].

Para el presente trabajo se utilizó el umbralado, en esta técnica se toma en cuenta un intervalo de tonalidad, los pixeles que tengan su valor dentro del intervalo se les asigna el valor más alto de luminosidad mientras que a los que se encuentren fuera se les asigna 0 ; quedando únicamente las formas que se encuentren en los intervalos.

\subsection{Lógica difusa}

La lógica fuzzy o lógica difusa es una técnica que permite simular el proceso del pensamiento humano a partir de incertidumbres o juicios de valor, por ejemplo "el sol es brillante" o "la noche es oscura". Esto lo logra utilizando como referencia sistemas basados en el conocimiento.

A diferencia de la lógica Booleana que considera la pertenencia absoluta o la no pertenencia de la variable a analizar con un conjunto, la lógica difusa toma en cuenta valores de pertenencia a dicho conjunto; por ejemplo: una persona que mide 1.70 en lógica booleana únicamente puede ser clasificada como alta o no alta, en cambio con lógica difusa la misma persona tiene un porcentaje de pertenencia a alta. Adicionalmente, la lógica difusa permite hacer relación de variables lingüísticas como se observó en el caso anterior con la palabra alto.

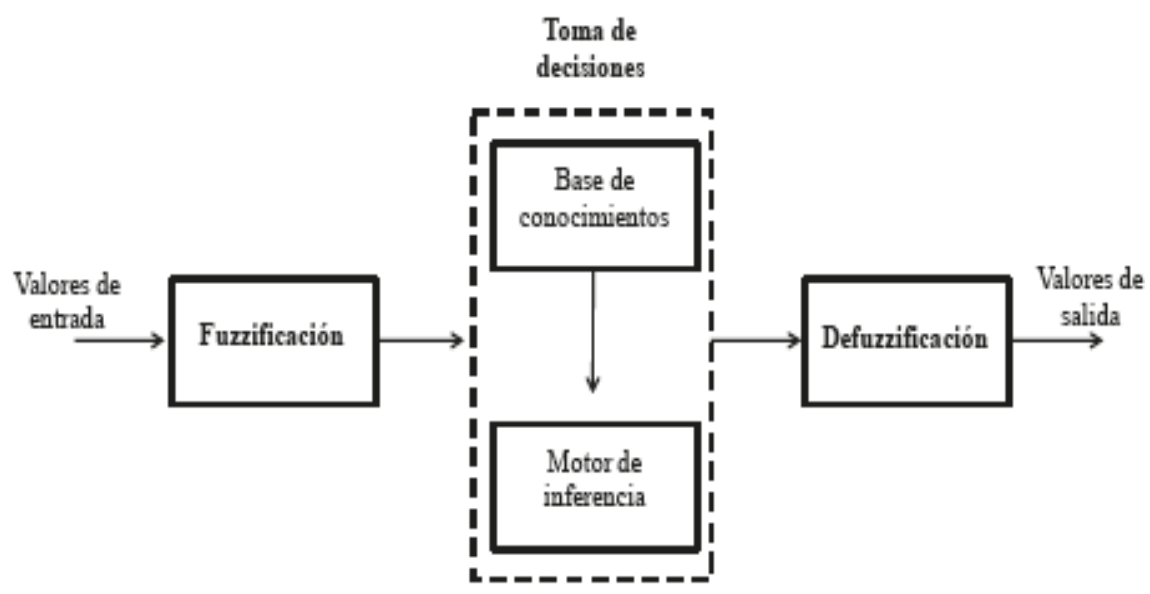

Fig. 3. Diagrama de bloques de un sistema de lógica difusa. 
Para lograr los valores de pertenencia, se utilizan variables de membresía, las cuales relacionan los valores que puede tener la variable con la pertenencia al conjunto. A partir de la función de membresía la variable pasa por un proceso de fuzzificación por el cual se obtiene el valor de pertenencia dentro de las funciones a las que llegue a pertenecer. Posteriormente se evalúa la entrada a partir de un conjunto de reglas que define el usuario, el resultado de esta evaluación pasa por un proceso de defuzzificación y se obtiene la salida del sistema (Fig. 3) [5].

\section{Metodología}

Este trabajo se desarrolló en tres etapas: captura de las imágenes, procesamiento de las imágenes y clasificación de la malformación. A continuación, se desarrollan cada una de ellas.

\subsection{Captura de la imagen}

Para capturar las imágenes se utilizaron cámaras web Logitech C170, con una resolución de 5 megapixeles; estas cámaras se colocaron en un andador construido con ángulo de hierro y plataforma de acrílico, posicionadas de manera que adquirieran la imagen de la planta de los pies del paciente que suba al dispositivo (Fig. 4).

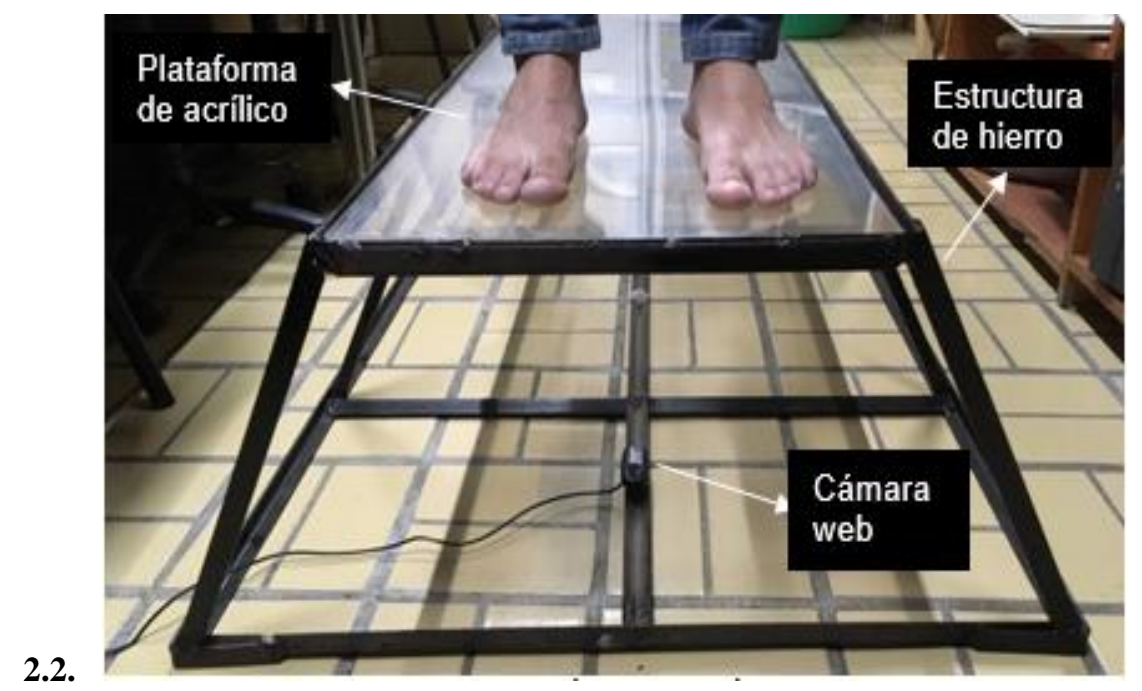

Fig. 4. Dispositivo para capturar fotografías de la planta del pie.

El control de las cámaras se realizó por medio de LabVIEW, configurando la cámara para capturar la fotografía en blanco y negro con una resolución de 8 bits (Fig. 5). 
Sistema de clasificación de deformaciones pédicas por procesamiento digital de imágenes ...

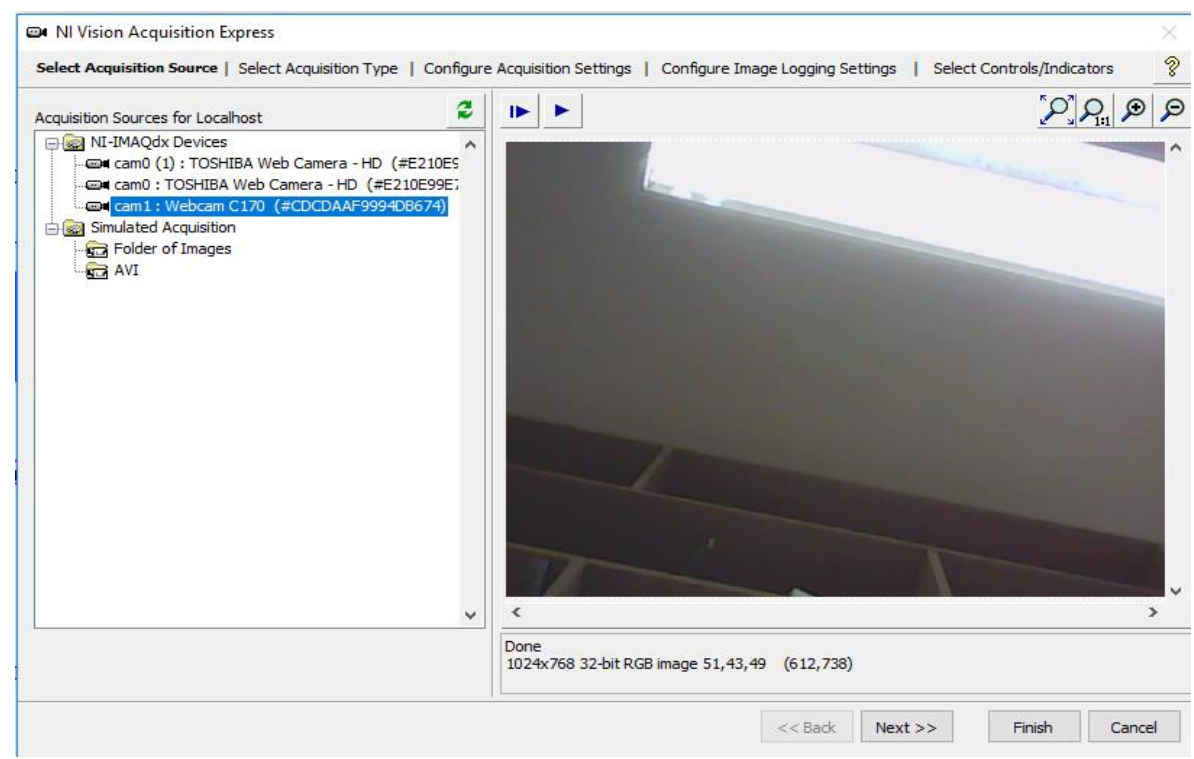

Fig. 5. Configuración de las webcams.

\subsection{Procesamiento de la imagen}

Después de obtener la imagen se le aplica un filtro de suavizado gaussiano para eliminar cualquier ruido generado en la captura de la imagen, este filtro es de comportamiento lineal, utilizando para éste una máscara $[0,1,0 ; 1,4,1 ; 0,1,0]$, adicionalmente se obtiene el histograma de la imagen para poder visualizar la variación de tonalidades en ella.

A partir del histograma se define el intervalo de intensidades de interés y se aplica umbralado por threshold permitiendo mantener la eliminación de los valores por arriba y abajo del umbral delimitando la planta del pie del resto de la imagen. La selección de la ventana de umbral es manual, a partir del histograma de la sección a umbralizar, se toma una media y se da una tolerancia

El proceso de umbralado se aplica siguiendo la ecuación 1, donde $g(x, y)$ es la función de intensidad de la imagen umbralada, $f(x, y)$ la función de intensidad de la imagen original y el intervalo $[a, b]$ como los valores de intensidad en los que se delimita la información de interés:

$$
g(x, y)= \begin{cases}255 \quad a \leq f(x, y) \leq b \\ 0 \text { c.o.c. }\end{cases}
$$

\subsection{Clasificación de la malformación}

Para empezar con la clasificación de la malformación se deben obtener medidas del pie, esto se logró mediante la toma de líneas rectas limitando el ángulo de detección en 80 a 110 grados evitando el contorno lateral del pie. Se genera una línea para el empeine 
y otra para el arco. Una vez que se obtienen las líneas se calcula su longitud mediante la ecuación de distancia entre dos puntos y se utiliza la ecuación 2, donde Le es la longitud del empeine y $L a$ la del arco para encontrar la relación entre las distancias antes mencionadas. Debido a que esta es un porcentaje, la distancia se obtiene en pixeles sin necesidad de convertir a otro sistema de medición:

$$
R=\frac{L e-L a}{L e} \times 100 .
$$

La relación obtenida es ingresada a un sistema de lógica difusa donde se clasifica el estado de malformación del pie. Para el diseño de las funciones de membresía se utilizó información de una guía de diagnóstico por podometría [2]. Se diseñaron siete funciones de entrada y cinco de salida a partir de esta información. Las funciones de salida se definieron como: plano, normal, cavo, cavo fuerte y cavo extremo (Fig. 6).

El proceso de defuzzificación se llevó a cabo mediante la técnica del centroide o centro del área. Esta técnica considera el área de las funciones de membresía con las que se empata la variable a analizar por las reglas del sistema difuso y las considera una sola. A partir de esto calcula el centro del área total obtenida, este centroide será la salida del sistema.

Se usaron siete reglas, siendo la respuesta del sistema la siguiente (Fig. 7) teniendo un valor cuantitativo de la malformación del pie.

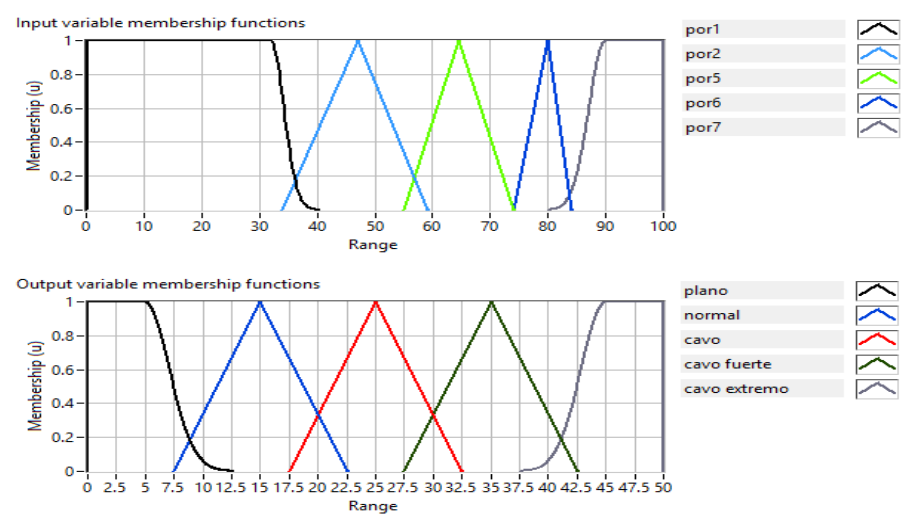

Fig. 6. Funciones de membresía del sistema de clasificación.

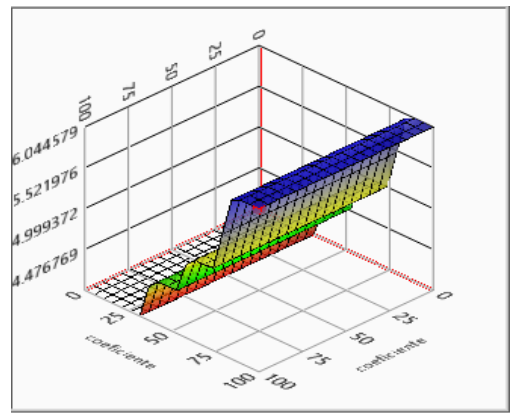

Fig. 7. Respuesta del sistema de lógica difusa. 
Sistema de clasificación de deformaciones pédicas por procesamiento digital de imágenes ...

\section{Resultados}

Se desarrolló un instrumento virtual para la clasificación de malformación pédicas en LabVIEW (Fig. 8) [6]. En éste se despliegan la imagen obtenida por la cámara, la imagen umbralizada, el histograma, la relación entre las medidas, la salida del fuzzy y la clasificación de la deformación.

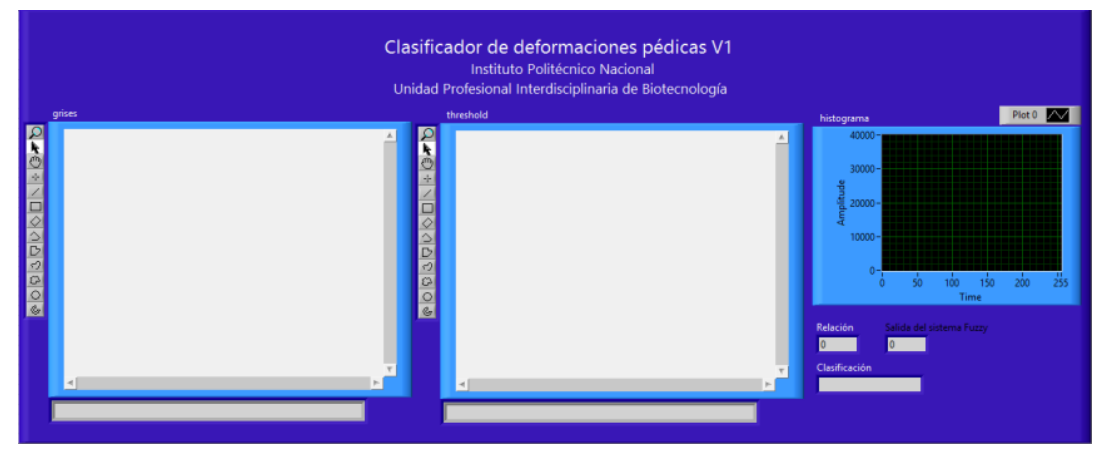

Fig. 8. Panel frontal del instrumento virtual.

Se obtiene la imagen mediante la cámara y se convierte de color a escala de grises (Fig. 9).

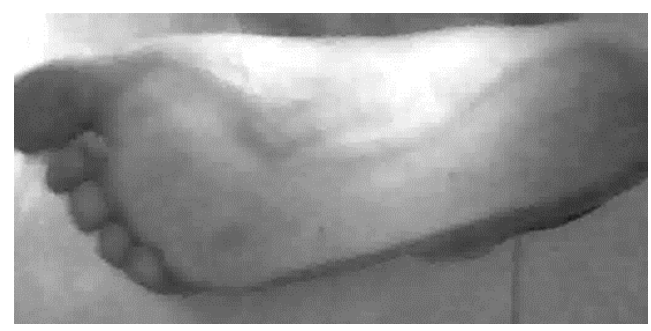

Fig. 9. Fotografía obtenida con el dispositivo de captura.

La imagen umbralizada se muestra a un costado, en esta se procura mantener la huella del pie (Fig. 10).

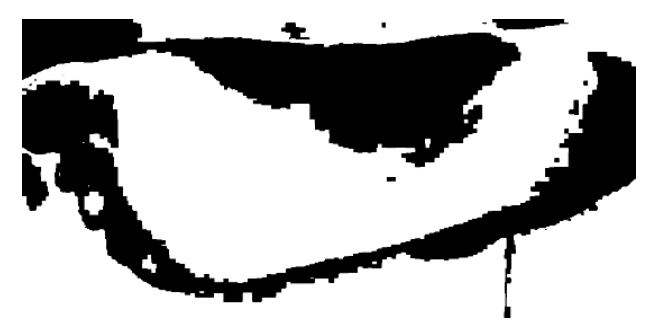

Fig. 10. Obtención de la huella mediante el umbralado. 
Finalmente se muestra en el panel frontal las líneas generadas para realizar la clasificación, el porcentaje de relación, el resultado del sistema Fuzzy y el tipo de malformación de la malformación obtenida (Fig. 11).

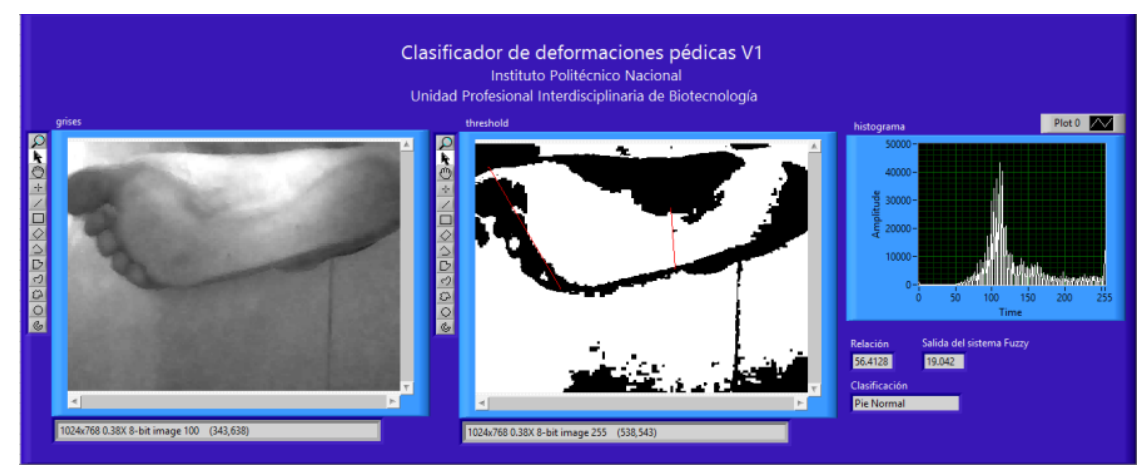

Fig. 11. Interfaz en funcionamiento.

Se realizaron pruebas con 10 sujetos diferentes con diagnóstico previo; cuatro de ellos sin malformación, dos con pie cavo y el restante con pie plano. Se muestran los resultados en la tabla 1 [7].

Tabla 1. Resultados de pruebas realizadas con el clasificador.

\begin{tabular}{c|c|c|c}
\hline Diagnóstico previo & $\begin{array}{c}\text { Resultado del } \\
\text { Programa }\end{array}$ & $\begin{array}{c}\text { \% de } \\
\text { relación }\end{array}$ & $\begin{array}{c}\text { Salida del } \\
\text { Fuzzy }\end{array}$ \\
\hline Pie normal & Pie normal & 56.41 & 19.04 \\
Pie normal & Pie normal & 52.76 & 14.99 \\
Pie normal & Pie normal & 50.76 & 15.01 \\
Pie normal & Pie normal & 52.86 & 14.98 \\
Pie cavo & Pie cavo & 66.51 & 25.04 \\
Pie cavo & Pie cavo & 72.69 & 25.12 \\
Pie cavo & Pie cavo fuerte & 82.07 & 35.32 \\
Ausencia de arco & Pie cavo extremo & 100 & 46.04 \\
Pie plano & Pie plano & 10.53 & 3.95 \\
Pie plano & Pie plano & 10.53 & 3.95 \\
\hline
\end{tabular}

\section{Conclusiones}

Se logró un sistema de clasificación de malformaciones pédicas, el cual selecciona entre cinco categorías ampliando el rango de clasificación con respecto a sistemas tradicionales. La resolución de las cámaras utilizadas fue suficiente para este sistema. El umbralado de la imagen ayuda al análisis al eliminar la información no deseada permitiendo el trazo de las líneas para la obtención de las medidas fundamentales. La determinación de las medidas fundamentales por medio de funciones lineales cumplió con las expectativas de la clasificación. El resultado de la clasificación se encuentra dentro de las variables de membresía del sistema Fuzzy para realizar la clasificación. La implementación del sistema de clasificación en un soporte permite que se expanda 
Sistema de clasificación de deformaciones pédicas por procesamiento digital de imágenes ...

el sistema para realizar análisis de la marcha. Todo esto, facilita el proceso de diagnóstico por parte del especialista.

\section{Referencias}

1. Silverman, M.: Ortopedia y Traumatología. Médica Panamericana. Argentina (2010)

2. Barrera, R., Siles, J. A., Concepción, L.: Aplicación didáctica para la valoración de un fotopodograma en las clases de educación física. Revista digital efdeportes No. 141, Argentina (2010)

3. Ready, S., Kwon K.: Practical guide to machine vision software. Wiley VCH. Singapur (2015)

4. Grimson, W. E. L., Huttenlocher, D. P.: Object recognition by computer: the role of geometric constraints (2015)

5. Ponce, P.: Inteligencia Artificial con Aplicaciones a la Ingeniería. Alfaomega, México (2010)

6. Lajara, J.R., Pelegri, J.: LabVIEW entorno gráfico de programación. Marcombo, España (2011)

7. Sanchez-Velarde, E., Sotelo de Avila, A. A., Cabrera-Llanos, I. A.: Clasificador fuzzytriage en sala de urgencias, aplicando máquina de estados en LabView. En: Memorias del VI Congreso Nacional de Tecnología Aplicada a Ciencias de la Salud, México: INAOE. (2015) 